\title{
Mediation Role of Health Service Use and Depression on Multimorbidity and Self-rated Health in Home-based Long-term Care Residents: a Cross- sectional Study
}

Huosheng Yan

Anhui Medical University https://orcid.org/0000-0003-4330-4373

Wenjia Peng

Bengbu Medical College

Mengying Li

Fudan University

Xinghui Li

Fudan University

Tingting Yang

Fudan University

Zijing Wang

Anhui Medical University

Cancan Li

Anhui Medical University

Xianjie Jia

Bengbu Medical College

Zhi Hu ( $\square$ aywghz@ahmu.edu.cn )

School of Health Service Management, Anhui Medical University https://orcid.org/0000-0001-54540422

Ying Wang

Fudan University

\section{Research}

Keywords: Multimorbidity, Self-rated health, Depression, Health Service Use, Mediation, Structural equation modeling

Posted Date: May 4th, 2021

DOl: https://doi.org/10.21203/rs.3.rs-446881/v1 
License: (c) (i) This work is licensed under a Creative Commons Attribution 4.0 International License. Read Full License 


\section{Abstract}

Background: Multimorbidity is common among older people and a major cause of reduced quality of life. The study aim was to investigate the relationship between multimorbidity and self-rated health and its mediators in home-based long-term care residents.

Methods: Participants were 1067 home-based long-term care residents covered by long-term care insurance in Shanghai. Stratified sampling was used to select participants from six Shanghai districts. Data were collected using face-to-face interviews. Multimorbidity was defined as co-occurrence of $\geq 2$ chronic diseases in the same person. The 30 -item Geriatric Depression Scale was used to assess depressive symptoms. Structural equation modeling was used for data analysis.

Results: The findings showed that $59.4 \%$ of participants had multimorbidity and $67.7 \%$ reported depressive symptoms. The mean self-rated health score was 1.97 (standard deviation [SD] = 0.861) and mean health service use frequency was $1.61(S D=3.406)$ per month. Compared with participants with no multimorbidity, those with multimorbidity were more likely to report low self-rated health $(\beta=-0.141$, $p<0.001)$, more severe depressive symptoms $(\beta=0.100, p<0.001)$, and more health service use $(\beta=0.121$, $p<0.001)$. Low self-rated health may be caused by depression and health service use $(\beta=-0.280$, $p<0.001)$. The effect of multimorbidity on self-rated health was significantly mediated by depression ( $\beta=$ $-0.024, p<0.001)$ and health service use $(\beta=-0.034, p<0.001)$.

Conclusion: Multimorbidity is associated with self-rated health, and depression and health service use mediate this association. Prevention and proper management of multimorbidity and depression in longterm care residents may help to maintain and improve quality of life.

\section{Introduction}

The global population is rapidly aging; the number of older adults ( $>60$ years) has been estimated as 1049 million in 2020 (13.4\% of the total global population). As one of the most rapidly aging countries in the world, China has an estimated 249 million older adults (> 60 years) in 2020 (17.3\% of the total population), and the number will reach 363 million in 2030 (24.8\% of the total population)[1]. However, increased life expectancy is not necessarily associated with better quality of life[2]; aging is generally associated with chronic multimorbidity[3]. Faced with an increase in the number of older adults and a decrease in quality of life, the Chinese Ministry of Human Resources and Social Security issued guidelines on June 27, 2016, about implementing a long-term care insurance (LTCl) policy in 15 pilot cities. The LTCl is a social insurance system that provides basic life care and daily nursing services to disabled and older adults[4]. Home-based long-term care is the main type of long-term care. It provides more than just illness care; it aims to enable individuals with multimorbidity or disabilities to live their lives as fully as possible[5].

Multimorbidity, the co-occurrence of two or more chronic physical conditions[6], is common among older people. As the global population ages, the prevalence of disease has been increasing[7]. A systematic 
review of the prevalence of multimorbidity found that $6.4-76.5 \%$ of individuals aged 60 years or more in China had multimorbidity[8]. The growing epidemic of multimorbidity has posed tremendous challenges, not only for individuals and their families[9], but also for public health policy and health care systems[10]. The growing prevalence of multimorbidity demands a greater understanding of its effects on overall patient health[6]. Compared with people with a single illness, people with multimorbidity require more complex management of their medical needs that should entail consideration of social and emotional functions[11]. In addition, multimorbidity is associated with a wide range of disabilities, functional decline, and poor quality of life[12].

Self-rated health is a subjective assessment of mental and physical wellness, and is associated with morbidity, mortality, and health care utilization $[13,14]$. For patients with a chronic disease and for longterm care residents, self-rated health tends to reflect reduced activities of daily living and long-term risk of mortality $[15,16]$. Chronic diseases affect people's ability to perform daily activities, and such individuals usually experience a decline in their quality of life $[17,18]$. Most studies on the association between selfrated health and multimorbidity focus on specific populations or specific diseases or disease groups. For example, among middle-aged and older adults, poorer self-rated health is strongly associated with single chronic diseases, particularly cardiovascular diseases, multimorbidity, and poor psychosocial function[19]. However, different groups may show differences in the validity of self-rated health as a predictor of mortality risk[20].

Factors that mediate the association between multimorbidity and self-rated health in long-term care residents have rarely been explored. Identification of such factors is essential to develop effective measures to improve self-rated health in this population. Depression and health service use may mediate the relationship between multimorbidity and self-rated health. Depression is a clinically significant and growing public health issue and the third leading cause of disability worldwide[21]. Multimorbidity may increase the risk of depression in elderly people through physiological, pharmacological, or psychological mechanisms[22], and may thus affect self-rated health[23]. Furthermore, individuals with multimorbidity report poorer self-rated health with certain disorders because of depression[20]. Multimorbidity and selfrated health are closely related to health service use. Long-term care residents showed higher proportions of multimorbidity and health service use[24]. In addition, both multimorbidity and depression increase the frequency of health service use[22, 25], and frequent health service use is usually accompanied by lower self-rated health[26].

Thus, in this population-based study, we aimed to a) explore the associations between multimorbidity and self-rated health among Chinese community-based long-term care residents, and b) test the extent to which the association between multimorbidity and low self-rated health is mediated by depression and health service use. We hope that our findings will contribute to improving the quality of life of elderly people and help them to respond positively to the aging process.

\section{Methods}




\section{Study Design and Population}

The analyses were carried out using data from a cross-sectional study conducted in Shanghai. Shanghai, on the eastern coast of China, is one of the LTCI policy pilot cities and is divided into 17 districts, which are further grouped into eight central urban areas, and nine suburbs[27]. Shanghai is an aging city; there are 5.18 million older adults (> 60 years) in the city (35.2\% of the total population). As a response to aging challenges, Shanghai introduced an LTCl program for elderly people in 2017. The program covers elderly people aged over 60 years who participate in public health insurance schemes, and it reimburses approximately $85-90 \%$ of health care costs[28].

Stratified sampling was used to select research subjects. We first selected three Shanghai LTCI pilot areas: Xuhui, Putuo, and Jingan districts. Then, considering the environmental, social, and economic differences between the centre, inner suburbs, and outer suburbs, we randomly selected Jinshan, Baoshan, and Songjiang districts from the above three areas. One street was selected from the six districts according to the stroke order of one Chinese character from the street name. Finally, one to three communities from the six streets were randomly selected and elderly people from those communities randomly selected according to their age. The exclusion criteria were (a) $<60$ years old; (b) not covered by Shanghai LTCl; (c) unable to cooperate with the investigator owing to severe cognitive impairment or hearing, language, expression, or other deficits. The present analysis used data from 1067 participants with complete data for the variables analyzed.

The survey was conducted by trained investigators who provided guidance to participants in the form of one-to-one discussions or help filling out the questionnaires. All participants voluntarily participated in the survey after providing their informed consent.

\section{Measurement}

\section{Multimorbidity}

We assessed the presence of 17 self-reported diseases or conditions by asking "Have you been diagnosed with the following conditions by a doctor": hypertension; diabetes or high blood sugar; cancer; heart disease; stroke; chronic lung disease; digestive disease; arthritis or rheumatism; intervertebral disk disease; chronic low back pain; eye disease; psychiatric disease; varicose veins; osteoporosis; cholecystitis; urinary tract stones; and anemia. Chronic lung diseases included medically diagnosed bronchitis, pneumonia, emphysema, chronic obstructive pulmonary disease, and tuberculosis. Eye diseases were defined as medically diagnosed cataract, glaucoma, or vision loss. Multimorbidity was defined as co-occurrence of two or more of the 17 chronic diseases in the same person [6].

\section{Self-rated health}


Self-rated health was measured using an item that was developed and used by the World Health Organization[29]. Participants were asked, "Generally speaking, in comparison with other people of your age, how do you feel about your physical condition?" Possible responses were very good, good, fair, poor, or very poor.

\section{Mediating Variables}

Depressive symptoms were assessed using the validated Chinese version of the 30-term Geriatric Depression Scale (GDS-30). The GDS-30 has been widely used in many countries and is an effective measure of depression in elderly people in long-term care[30, 31]. Each scale item is a question with the possible responses of "yes" or "no." The total score ranges from 0 to 30 points; scores $\geq 11$ are considered to indicate depressive symptoms. The GDS-30 has shown high sensitivity and specificity with older Chinese samples, and Cronbach's a is 0.890 , indicating good internal consistency[32].

Health service use was measured using three indicators: average number of outpatient visits per month in the last year, number of hospitalizations in the last year, and average number of emergency department visits per month in the last year. The combined frequency of these three indicators was used as the measure of health service use.

\section{Covariates}

To control potential confounding variables, age (years), gender, and income (yuan/per month) were examined as adjustment variables.

\section{Statistical Analysis}

Descriptive statistics were used to summarize the distribution of participant characteristics, categorical variable frequencies (percentages), and the means of continuous variables (standard deviation, SD).

Structural equation modeling was used to investigate the associations between multimorbidity, self-rated health, and potential mediators. This is an excellent statistical technique to evaluate a priori models, identify mediators, and elucidate direct and indirect paths between variables. Multimorbidity was treated as an exogenous variable and used to estimate the following two variables: the latent variable (depression) and the observed variable (health service use) (Fig. 1). The maximum likelihood method was used to estimate model parameters. The model fit was estimated using the following indicators: (a) ratio of chi-square value to degrees of freedom $\left(\chi^{2} / d f\right) \leq 3$; (b) comparative fit index (CFI) $\geq 0.90$; (c) standardized root mean square residual $(S R M R)<0.05$; (d) root mean square error of approximation $($ RMSEA $)<0.05[33]$. Descriptive analyses were performed using SPSS 25.0 Statistics for Windows, version 25.0 (IBM Corp, Armonk, NY, USA) and Mplus, version 7.4 (Muthen \& Muthen, Los Angeles, CA, USA) was used for the structural equation modeling.

\section{Results}

\section{Characteristics of the Study Participants}


For the 1067 participants, the mean age was $82.33(S D=8.010)$ years, the mean income was 3141.50 $(\mathrm{SD}=2.379$ ) yuan per month, and $58.8 \%$ were women. Of participants, $19.0 \%$ had no chronic disease, and $21.6 \%, 23.8 \%, 19.6 \%$, and $16.0 \%$ had one, two, three, and four or more chronic diseases, respectively. The average number of chronic diseases per participant was 2.05 (SD $=1.648) ; 59.4 \%$ of participants had multimorbidity and $67.7 \%$ had depression. The mean self-rated health score was $1.97(S D=0.861)$ (the total possible score was 5$)$. The mean frequency of health service use was $1.61(S D=3.406)$ per month (Table 1).

Table 1

Baseline characteristics of participants

\begin{tabular}{|c|c|c|}
\hline Variables & Mean (SD) & $\mathrm{N}(\%)$ \\
\hline Age & 82.33(8.0) & \\
\hline \multicolumn{3}{|l|}{ Gender } \\
\hline Male & & $440(41.2)$ \\
\hline Female & & $627(58.8)$ \\
\hline Income & $3141.50(2.4)$ & \\
\hline \multicolumn{3}{|l|}{ Chronic conditions } \\
\hline Numbers of chronic & $2.05(1.7)$ & \\
\hline No & & 203(19.0) \\
\hline 1 & & $230(21.6)$ \\
\hline 2 & & $254(23.8)$ \\
\hline 3 & & $209(19.6)$ \\
\hline$\geq 4$ & & $171(16.0)$ \\
\hline \multicolumn{3}{|l|}{ Multimorbidity } \\
\hline Yes & & $634(59.4)$ \\
\hline No & & $433(40.6)$ \\
\hline \multicolumn{3}{|l|}{ Depression } \\
\hline No & & $345(32.3)$ \\
\hline Yes & & $722(67.7)$ \\
\hline Self-rated health & $1.97(0.9)$ & \\
\hline Health service use & $1.61(3.4)$ & \\
\hline
\end{tabular}


Of the 17 chronic diseases examined, hypertension (58.58\%) was the most common condition, followed by heart disease (38.61\%) and diabetes (22.68\%) (Fig. 2).

\section{Structural Equation Modeling}

A structural equation model was built to estimate the relationships between multimorbidity, self-rated health, depression, and health service use (Fig. 1). The model fit indices, the standardized direct effects of four variables, and the indirect effects of two mediation variables are shown in Table 2 and Table 3 after adjusting for age, gender, and income. The model fit indices were as follows: $\chi^{2} / d f=2.185, \mathrm{CFI}=0.987$, SRMR $=0.023, \mathrm{RMSEA}=0.033$, and indicate that the model had a good fit.

Table 2

Model fit indices

\begin{tabular}{|ll|}
\hline Model Fit & Index \\
\hline$\chi^{2} / \mathrm{df}$ & 2.185 \\
\hline CFI & 0.987 \\
\hline SRMR & 0.023 \\
\hline RMSEA & $0.033(0.020,0.046)$ \\
\hline $\begin{array}{l}\text { CFI: confirmatory fit index; SRMR: standardized root mean square residual; RMSEA: root mean square } \\
\text { error of approximation. }\end{array}$ \\
\hline
\end{tabular}


Table 3

Standardized direct and indirect effects

\section{Variables}

Standardized coefficient

Direct effects

Self-rated health

Multimorbidity

$-0.141(-0.197,-0.086)$ **

Depression

$-0.235(-0.295,-0.162)$ **

Health service use

$-0.280(-0.333,-0.229)$ **

\section{Depression}

Multimorbidity

$0.100(0.039,0.155)$ **

Health service use

$0.075(0.014,0.130)$ **

\section{Health service use}

Multimorbidity

$0.121(0.061,0.181)$ **

\section{Indirect effects}

Multimorbidity ->Depression ->Self-rated health

$-0.024(-0.044,-0.005) * *$

Multimorbidity $->$ Health service use $\rightarrow$ Self-rated health

$-0.034(-0.059,-0.011) * *$

Multimorbidity $->$ Depression $->$ Health service use $\rightarrow$ Self-rated health

$-0.002(-0.005,0.000)$ *

Total Indirect

$-0.141(-0.216,-0.069)$ **

${ }^{\star \star} p<0.001,{ }^{*} p<0.05$; adjusted for age, gender, and income.

\section{Relationships Among the Variables}

The model showed that multimorbidity had a significant direct effect on self-rated health $(\beta=-0.141$, $95 \% \mathrm{Cl}=-0.197$ to -0.086$)$; participants with multimorbidity were more likely to report low self-rated health.

Multimorbidity was significantly associated with two mediating variables. Participants with multimorbidity were more likely to have depression symptoms $(\beta=0.100,95 \% \mathrm{Cl}=0.039$ to 0.155$)$ than those who did not have multimorbidity. Multimorbidity was also associated with more frequent health service use $(\beta=0.121,95 \% \mathrm{Cl}=0.061$ to 0.181$)$.

Associations were also found between self-rated health and the two mediating variables. Participants with higher GDS-30 scores had lower self-rated health $(\beta=-0.235,95 \% \mathrm{Cl}=-0.295$ to -0.162$)$. In 
addition, self-rated health was significantly influenced by health service use $(\beta=-0.280,95 \% \mathrm{Cl}=-0.333$ to - 0.229); participants with lower health service use were more likely to report greater self-rated health.

Furthermore, the association between the two mediating variables of depression and health service use was significant: frequent use of health services was associated with greater depression ( $\beta=0.075,95 \%$ $\mathrm{Cl}=0.014$ to 0.130 ). The direct effect and the indirect effect were significant both before and after the covariates were added to the model. (Table 3).

\section{Mediating Role of Depression and Health Service Use}

The multiserial mediator model demonstrated that depression plays a potentially mediating role in the association between multimorbidity and self-rated health $(\beta=-0.024,95 \% \mathrm{Cl}=-0.044$ to -0.005$)$. Health service use also showed a mediating effect on the relationship between multimorbidity and self-rated health $(\beta=-0.034,95 \% \mathrm{Cl}=-0.059$ to -0.011$)$. In addition, the indirect effect of multimorbidity on selfrated health, via depression and health service use, was significant. Of the three potential mediating pathways, health service use had a stronger effect than the other pathways.

\section{Discussion}

This study confirmed the correlation between multimorbidity and self-rated health in home-based longterm care residents: residents with multimorbidity were more likely to report low self-rated health. In addition to a direct association between multimorbidity and self-rated health, there was an indirect effect of multimorbidity on self-rated health through three potential pathways: via depression, via health service use, and via depression and health service use.

The results showed that $59.4 \%$ of home-based long-term care residents had multimorbidity, which is lower than the percentage of older people with multimorbidity in a previous longitudinal nationwide survey in China[34]. However, the difference may be a result of regional and participant differences. The mean self-rated health score in this study was 1.97, indicating that the health of home-based long-term care residents is poor. This result is the same as that of a previous study of long-term care residents in China[16]. We also found that $67.7 \%$ of home-based long-term care residents had depression, higher than in a previous study conducted with Chinese older adults[30]. This difference may reflect the poor physical condition of long-term care residents.

To our knowledge, this is the first population-based study of Chinese home-based long-term care residents to investigate the relationship between multimorbidity and self-rated health and the mediating role of health service use and depression. Previous studies have identified a correlation between multimorbidity and low self-rated health[17-19]. However, these studies have used different types of participants (e.g., older people in the community or in health facilities) or analytical methods (e.g., traditional regressions and correlations). Furthermore, the chronic diseases these studies focused on are frequently occurring diseases in the region. Although these studies differ in specific details, their findings 
regarding the negative effect of multimorbidity on self-evaluated health are consistent, and are supported by the present findings.

We found that multimorbidity was associated with an increased likelihood of depression in home-based long-term care residents. One study conducted with community-dwelling elderly people in eastern China indicated that people with multimorbidity were more likely to have depressive symptoms (odds ratio = $1.42 ; 95 \% \mathrm{Cl}=1.19$ to 1.70 )[35]. Previous research has suggested that some chronic diseases like diabetes, stroke, and thyroid disorders cause or contribute to depression by causing pathophysiological changes in the brain, endocrine system, or immune system[36,37]. Our finding of a correlation between depression and self-rated health supports previous survey evidence of this association in African American men[20]. A study conducted in older people living in Shanghai found that both chronic disease and depression are predictors of self-rated health[22], and concluded that some depressive symptoms and depression-related negative emotions may lead elderly people to perceive their health negatively, thereby reducing their health satisfaction. These are possible explanations of our finding that depression mediates the association between multimorbidity and self-rated health. Some chronic diseases cause depression through pathophysiological changes, and multimorbidity aggravates depression symptoms (worse depression is associated with lower self-rated health).

We found that home-based long-term care residents with multimorbidity use health services more frequently, which has not previously been confirmed for long-term care residents, although previous studies on retired Chinese participants[38] and Canadian home care clients with dementia[39] have found such an association. Multimorbidity is associated with a wide range of disabilities and functional decline, which increase the demand for medical services. Unlike long-term health facility residents who have professional caregivers available 24 hours a day[40], people receive home-based long-term care services at a fixed time. This may explain the association between multimorbidity and health service use in homebased long-term care residents. This study showed an association between health service use and selfrated health, which reflects previous findings from Singapore[41]. Frequent health service use is often a result of poor health status, which is also the main reason for low self-rated health. In addition, health service use was also associated with depression in this study, indicating that depression may predict health service use to some extent. In a study on American older adults, depressive symptoms were positively related to health service use[42], which is consistent with our results. A possible reason for this association is that depressive symptoms lead older people to have a negative perception of their health, thereby increasing their use of health services. We found a mediating role of health service use and a multiserial mediation pathway by which depression and health service use affect the association between multimorbidity and self-rated health. These mediation pathways have been discussed in previous research[18,25]. Multimorbidity may cause disability, functional decline, and depression and may increase the demand for medical services. Frequent health service use may lead elderly people to perceive their health negatively, thus reducing their self-rating of health.

There were several study limitations. First, there was a higher proportion of females (58.8\%) than of males. This may be because individuals covered by LTCI are older; in this study, the mean age of 
participants was 82.33 years. Second, a small number of participants with consciousness deficits were excluded, resulting in a slight decline in the efficiency of the questionnaire. Finally, the findings cannot be generalized to elderly people in long-term care facilities, and therefore cannot be used to provide guidance on improving the quality of life of elderly people in long-term care. These limitations need to be addressed in future research.

\section{Conclusions}

The findings indicate that multimorbidity can aggravate depressive symptoms, increase the utilization of health services, and result in poor self-rated health. Furthermore, depression and health service use mediate the association between multimorbidity and poor self-rated health. This study explored possible pathways between multimorbidity and self-rated health. The findings may have implications for the proper management of chronic diseases, self-rated health, depression, and health service use. In the long term, our research findings may help to improve long-term care in elderly people with chronic diseases. Multimorbidity conditions, depressive symptoms, and access to health services should be assessed and considered when developing health management plans for long-term care residents. In addition, depressive symptoms and health service use frequency should be taken into consideration in efforts to prevent poor self-rated health in long-term care residents with multimorbidity.

\section{Abbreviations}

LTCl: Long-term care insurance

\section{Declarations}

\section{Ethics approval and consent to participate}

The study was approved by the Fudan University Research Ethics Committee. (IRB\# 2020-07-0840). Written informed consent was provided by all participants prior to answering the questionnaire, including consent for de-identified data to be used in publications arising from the research.

\section{Consent for publication}

Not applicable.

\section{Availability of data and materials}

The datasets used and/or analysed during the current study are available from the corresponding author on reasonable request.

\section{Competing interests}

The authors declare that they have no competing interests. 


\section{Funding}

This work was supported by the National Natural Science Foundation of China (No.71974034) and the Anhui Health Development Strategy Research Center (No.0503011216). The views expressed are those of the authors. The funder has no role in the conduct of the research or the decision to submit this report.

\section{Authors' contributions}

Study concept and design: Y.W., Z.H. and X.J.; acquisition of data: M.L. X.L. and T. Y.; analysis and interpretation of data: H.Y., W.P., Z.W. and C.L.; Drafting of the manuscript: H.Y..

\section{Acknowledgements}

The authors are grateful to all participants who voluntarily agreed to complete the survey.

\section{References}

1. UN. World Population Prospects 2019: Highlights. New York, NY, USA: United Nations Department for Economic and Social Affairs; 2019.

2. Murray CJ, Barber RM, Foreman KJ, Abbasoglu Ozgoren A, Abd-Allah F, Abera SF, et al. Global, regional, and national disability-adjusted life years (DALYs) for 306 diseases and injuries and healthy life expectancy (HALE) for 188 countries, 1990-2013: quantifying the epidemiological transition. Lancet. 2015;386(10009):2145-91.

3. Beard JR, Officer A, de Carvalho IA, Sadana R, Pot AM, Michel JP, et al. The World report on ageing and health: a policy framework for healthy ageing. Lancet. 2016;387(10033):2145-54.

4. Zhang Y, Yu X. Evaluation of Long-Term Care Insurance Policy in Chinese Pilot Cities. Int J Environ Res Public Health. 2019;16(20).

5. Group WS. Home-based long-term care. World Health Organ Tech Rep Ser. 2000;898:i-v, 1-43.

6. Read JR, Sharpe L, Modini M, Dear BF. Multimorbidity and depression: A systematic review and metaanalysis. J Affect Disord. 2017;221:36-46.

7. Lutz W, Sanderson W, Scherbov S. The coming acceleration of global population ageing. Nature. 2008;451(7179):716-9.

8. Hu X, Huang J, Lv Y, Li G, Peng X. Status of prevalence study on multimorbidity of chronic disease in China: systematic review. Geriatr Gerontol Int. 2015;15(1):1-10.

9. Yarnall AJ, Sayer AA, Clegg A, Rockwood K, Parker S, Hindle JV. New horizons in multimorbidity in older adults. Age Ageing. 2017;46(6):882-8.

10. Kim KI, Lee JH, Kim CH. Impaired health-related quality of life in elderly women is associated with multimorbidity: results from the Korean National Health and Nutrition Examination Survey. Gend Med. 2012;9(5):309-18.

11. Roland M, Paddison C. Better management of patients with multimorbidity. Bmj. 2013;346:f2510. 
12. Marengoni A, Angleman S, Melis R, Mangialasche F, Karp A, Garmen A, et al. Aging with multimorbidity: a systematic review of the literature. Ageing Res Rev. 2011;10(4):430-9.

13. Jylhä M. What is self-rated health and why does it predict mortality? Towards a unified conceptual model. Soc Sci Med. 2009;69(3):307-16.

14. Wu S, Wang R, Zhao Y, Ma X, Wu M, Yan X, et al. The relationship between self-rated health and objective health status: a population-based study. BMC Public Health. 2013;13:320.

15. Wennberg P, Rolandsson O, Jerdén L, Boeing H, Sluik D, Kaaks R, et al. Self-rated health and mortality in individuals with diabetes mellitus: prospective cohort study. BMJ Open. 2012;2(1):e000760.

16. Xu R, Zhou X, Cao S, Huang B, Wu C, Zhou X, et al. Health Status of the Elderly and Its Influence on Their Activities of Daily Living in Shangrao, Jiangxi Province. Int J Environ Res Public Health. 2019;16(10).

17. Ishizaki T, Kobayashi E, Fukaya T, Takahashi Y, Shinkai S, Liang J. Association of physical performance and self-rated health with multimorbidity among older adults: Results from a nationwide survey in Japan. Arch Gerontol Geriatr. 2019;84:103904.

18. Sheridan PE, Mair CA, Quiñones AR. Associations between prevalent multimorbidity combinations and prospective disability and self-rated health among older adults in Europe. BMC Geriatr. 2019;19(1):198.

19. Marques A, Peralta M, Gouveia É R, Chávez FG, Valeiro MG. Physical activity buffers the negative relationship between multimorbidity, self-rated health and life satisfaction. J Public Health (Oxf). 2018;40(3):e328-e35.

20. Assari S, Smith J, Bazargan M. Depression Fully Mediates the Effect of Multimorbidity on Self-Rated Health for Economically Disadvantaged African American Men but Not Women. Int J Environ Res Public Health. 2019;16(10).

21. Gunn JM, Ayton DR, Densley K, Pallant JF, Chondros P, Herrman HE, et al. The association between chronic illness, multimorbidity and depressive symptoms in an Australian primary care cohort. Soc Psychiatry Psychiatr Epidemiol. 2012;47(2):175-84.

22. Chen $Y$, While AE, Hicks A. Self-rated health and associated factors among older people living alone in Shanghai. Geriatr Gerontol Int. 2015;15(4):457-64.

23. Park LT, Zarate CA, Jr. Depression in the Primary Care Setting. N Engl J Med. 2019;380(6):559-68.

24. von Berenberg P, Dräger D, Zahn T, Neuwirth J, Kuhlmey A, Gellert P. Chronic conditions and use of health care service among German centenarians. Age Ageing. 2017;46(6):939-45.

25. Han KM, Ko YH, Yoon HK, Han C, Ham BJ, Kim YK. Relationship of depression, chronic disease, selfrated health, and gender with health care utilization among community-living elderly. J Affect Disord. 2018;241:402-10.

26. Ho SH. Correlations Among Self-Rated Health, Chronic Disease, and Healthcare Utilization in Widowed Older Adults in Taiwan. J Nurs Res. 2018;26(5):308-15. 
27. Liu Y, Fei X, Zhang Z, Li Y, Tang J, Xiao R. Identifying the sources and spatial patterns of potentially toxic trace elements (PTEs) in Shanghai suburb soils using global and local regression models. Environ Pollut. 2020;264:114171.

28. Feng J, Wang Z, Yu Y. Does long-term care insurance reduce hospital utilization and medical expenditures? Evidence from China. Soc Sci Med. 2020;258:113081.

29. Skevington SM, Lotfy M, O'Connell KA. The World Health Organization's WHOQOL-BREF quality of life assessment: psychometric properties and results of the international field trial. A report from the WHOQOL group. Qual Life Res. 2004;13(2):299-310.

30. Rong J, Chen G, Wang X, Ge Y, Meng N, Xie T, et al. Correlation Between Depressive Symptoms And Quality Of Life, And Associated Factors For Depressive Symptoms Among Rural Elderly In Anhui, China. Clin Interv Aging. 2019;14:1901-10.

31. Jongenelis K, Pot AM, Eisses AM, Gerritsen DL, Derksen M, Beekman AT, et al. Diagnostic accuracy of the original 30-item and shortened versions of the Geriatric Depression Scale in nursing home patients. Int J Geriatr Psychiatry. 2005;20(11):1067-74.

32. Chan AC. Clinical validation of the Geriatric Depression Scale (GDS): Chinese version. J Aging Health. 1996;8(2):238-53.

33. Hu Lt, Bentler PM. Cutoff criteria for fit indexes in covariance structure analysis: Conventional criteria versus new alternatives. Structural Equation Modeling: A Multidisciplinary Journal. 1999;6(1):1-55.

34. Zhao YW, Haregu TN, He L, Lu S, Katar A, Wang H, et al. The effect of multimorbidity on functional limitations and depression amongst middle-aged and older population in China: a nationwide longitudinal study. Age Ageing. 2020.

35. You L, Yu Z, Zhang X, Wu M, Lin S, Zhu Y, et al. Association Between Multimorbidity and Depressive Symptom Among Community-Dwelling Elders in Eastern China. Clin Interv Aging. 2019;14:2273-80.

36. Evans DL, Charney DS, Lewis L, Golden RN, Gorman JM, Krishnan KR, et al. Mood disorders in the medically ill: scientific review and recommendations. Biol Psychiatry. 2005;58(3):175-89.

37. Penninx BW, Beekman AT, Ormel J, Kriegsman DM, Boeke AJ, van Eijk JT, et al. Psychological status among elderly people with chronic diseases: does type of disease play a part? J Psychosom Res. 1996;40(5):521-34.

38. Zhao Y, Atun R, Oldenburg B, McPake B, Tang S, Mercer SW, et al. Physical multimorbidity, health service use, and catastrophic health expenditure by socioeconomic groups in China: an analysis of population-based panel data. Lancet Glob Health. 2020;8(6):e840-e9.

39. Mondor L, Maxwell CJ, Hogan DB, Bronskill SE, Gruneir A, Lane NE, et al. Multimorbidity and healthcare utilization among home care clients with dementia in Ontario, Canada: A retrospective analysis of a population-based cohort. PLoS Med. 2017;14(3):e1002249.

40. Hong S, Yu P, Chen X, Qian S. Long-term care services and care workers in Hangzhou City, China: A cross-sectional survey. J Nurs Manag. 2019;27(7):1546-53.

41. George PP, Heng BH, De Castro Molina JA, Wong LY, Wei Lin NC, Cheah JT. Self-reported chronic diseases and health status and health service utilization-results from a community health survey in 
Singapore. Int J Equity Health. 2012;11:44.

42. Jiang L, Sun F, Zhang W, Wu B, Dong X. Health Service Use Among Chinese American Older Adults: Is There a Somatization Effect? J Am Geriatr Soc. 2019;67(S3):S584-s9.

Figures

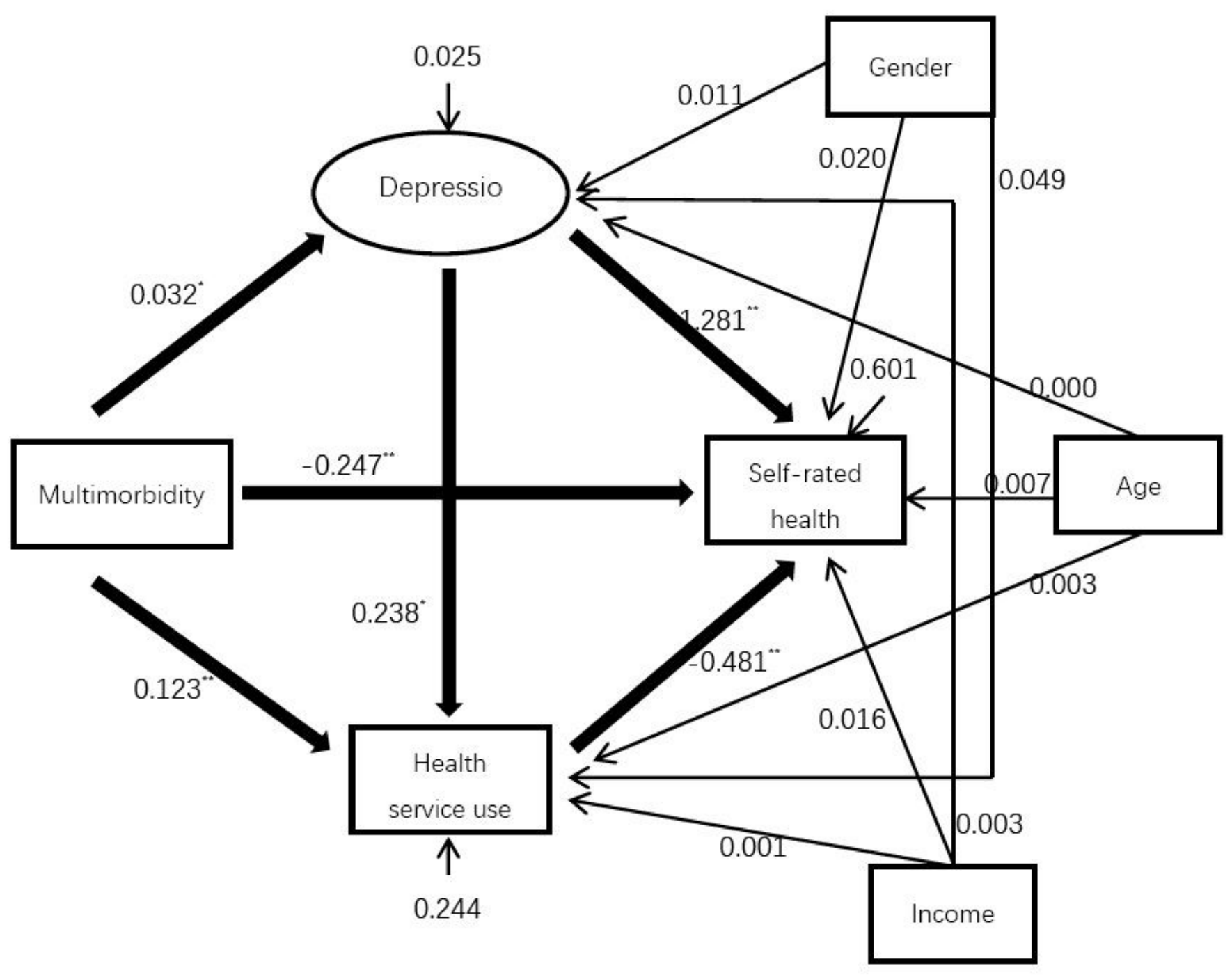

Figure 1

Results of the structural equation model. ${ }^{\star \star} p<0.001,{ }^{*} p<0.05$; adjusted for age, gender, and income. 


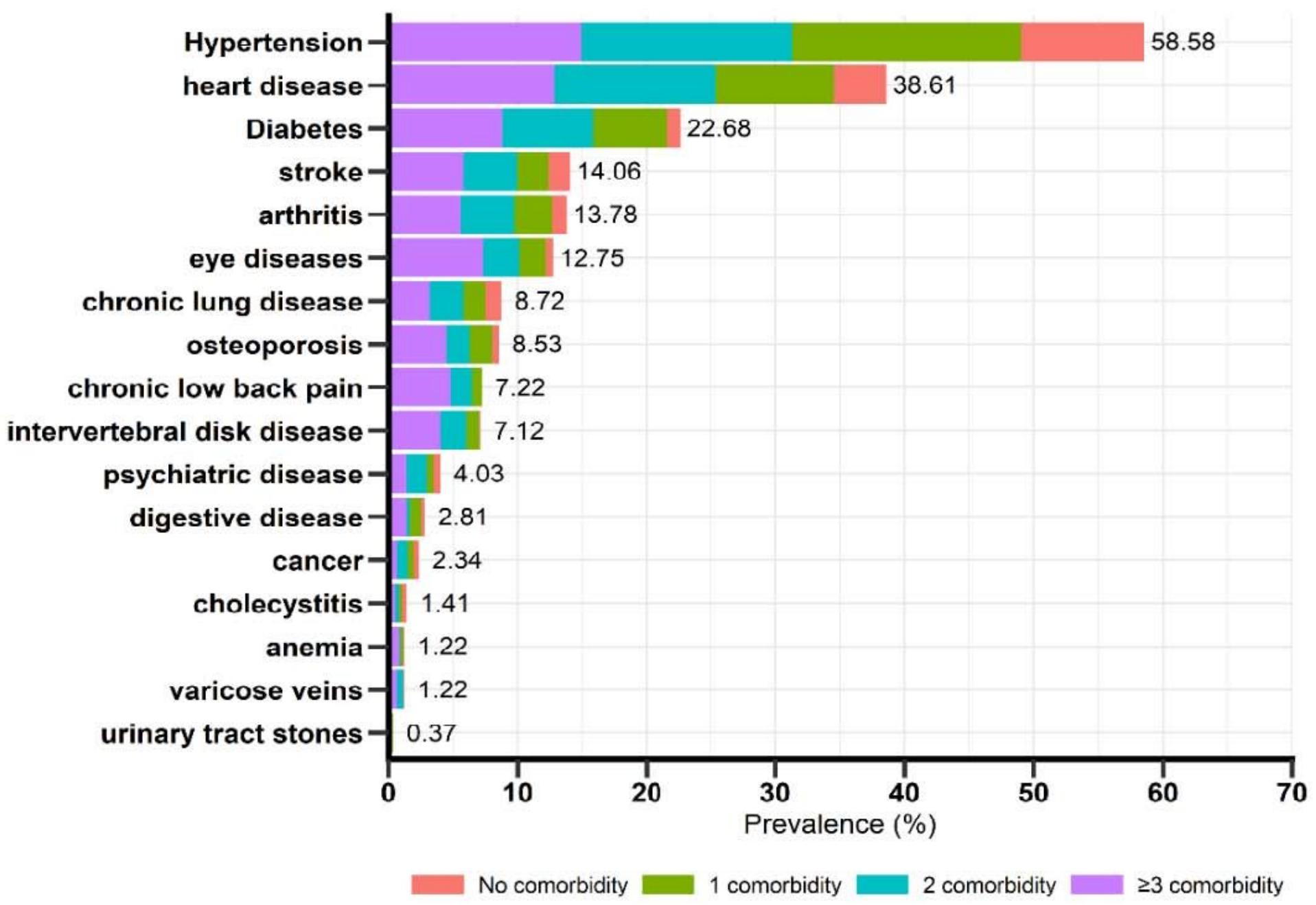

Figure 2

Prevalence (per 100 population) and multimorbidity $(\mathrm{N}=1067)$. 